\title{
Disparate Body Composition Outcomes for Underwater Weighing, Dual-Energy X-Ray Absorptiometry, and Two Bioelectrical Impedance Devices in Overweight Young Adults
}

\author{
Michelle Alencar $^{1 *}$, Kelly E. Johnson ${ }^{2}$, Nathan Cole ${ }^{2}$, Ann L. Gibson ${ }^{2}$ \\ ${ }^{1}$ Department of Kinesiology, California State University Long Beach, Long Beach, CA \\ ${ }^{2}$ Department of Health, Exercise, and Sports Sciences, University of New Mexico, Albuquerque, NM
}

Received: : December 09, 2016; Accepted: January 02, 2017; Published: January 19, 2017

*Corresponding author: Michelle Alencar, Department of Kinesiology, California State University, Long Beach, 1250 Bellflower Blvd. University, Long Beach, CA 90840-4901, Tel: 562-985-1435; E-mail: michelle.alencar@csulb.edu

\begin{abstract}
Introduction: As the prevalence of obesity rises, so does the need for reliable Body Composition (BC) methods. Many methods of $\mathrm{BC}$ analysis exist, however validity between methods has been questioned. Uncertainty exists within the scientific literature about which laboratory method and field measurement is applicable for the overweight population to estimate Percent Body Fat (\% BF) There is scarce research comparing Hydrostatic Weighing (HW) and Dual Energy X-Ray Absorptiometry (DXA) with field methods in overweight individuals. The purpose of this study was to compare HW and DXA with Multi-Frequency (MF-BIA) and a Single-Frequency (SFBIA) Bioelectrical Impedance (BIA) device.
\end{abstract}

Methods: Twenty-five overweight and obese adults (BMI $30.1 \pm$ $4.1 \mathrm{~kg} / \mathrm{m}^{2}, 13$ males, 12 females, ages $24.6 \pm 4.7 \mathrm{y}$ ) were randomly assessed by vertical SF-BIA, and supine MF-BIA against DXA and lastly HW. All participants followed standardized pre-testing guidelines for BC assessment. Agreement was assessed using a 2 (group) x 4 (method) RMANOVA and post-hoc t-tests were applied as appropriate. All methods of body composition were used and compared to DXA and HW.

Results: Significant main effects between $\mathrm{BC}$ methods were observed $F(1,23)=63.8, p<0.001)$. Where $\% \mathrm{BF}$ values were higher using the DXA $(34.2 \pm 10.6 \%)$ vs. HW $(28.2 \pm 9.3 \%)(p<0.001)$. Additionally, \% BF values were higher using the MF-BIA ( $30.9 \pm 58.8$ $\%)$ compared to the SF-BIA $(26.2 \pm 8.0 \%)(p<0.001)$. \%BF values were significantly correlated between SF-BIA and HW $(p<0.05, r=$ $0.902)$ as well as between MF-BIA and DXA $(p<0.05, r=0.709)$.

Conclusions: Differences in \%BF were evident when comparing the vertical SF-BIA and supine MF-BIA with HW and DXA. The field method SF-BIA was found to correlate with the laboratory method HW. Whereas the field method MF-BIA was found to correlate with DXA.

Keywords: Fat Mass; Fat-Free Mass; Percent Body Fat

\section{Introduction}

The well-documented rise of overweight and obesity rates in the United States represents a major public health concern, with recent reports estimating that an astonishing $16.9 \%$ of juvenile
Americans, and $34.9 \%$ of American adults, are obese [1].This unfortunate prevalence of obesity, with associated long-term health consequences, places the precision and accuracy of the Body Composition Analysis (BCA) techniques used to estimate percent body fat $(\% \mathrm{BF})$ in a position of elevated clinical and practical importance, particularly for overweight or obese individuals [2]. Accurate and reliable in vivo determination of whole-body composition across a diverse array of subpopulations is critical to the veracity of subsequent diagnoses and prognostication, as well as crucial for dependable comparison of repeated analyses, often used to assess fluctuations in body composition over time, as with monitoring periods of anticipated weight loss or weight gain [2]. A variety of BCA methods exist, including. Hydrostatic Weighing (HW), Dual-Energy X-Ray Absorptiometry (DXA), Air Displacement Plethysmography (ADP), Skin Fold Measurement (SF), Bioelectrical Impedance Analysis (BIA) and Ultrasound (US); each presenting individual virtues and limitations [3-6].

The early stages of BCA were largely predominated by HW, or hydro densitometry, which was regarded as the "gold standard" laboratory BCA technique for many decades $[5,6]$. Based on the Archimedes principle, HW compares subjects' dry weight with underwater weight to establish body volume and determine density [7]. This method is comparatively time consuming, and can be difficult or uncomfortable for some subjects, as they are required to perform repeated maximal voluntary exhalations while completely submerged [5, 8]. HW is also limited by several inherent sources of error, these largely stem from variability in residual airway volume [5], and the dependence of this method on a number of static reference values, drawn from limited animal and cadaver research performed in the 1940's and 50's [9-11]. These include static assumptions regarding the densities of Fat Mass (FM) (0.9007 g/ $\mathrm{cm}^{3}$ ), and Fat-Free Mass (FFM) (1.100 g/ $\mathrm{cm}^{3}$ ), as well as the water content of FFM (73.2\%), which are often uniformly applied to all subjects irrespective of age, ethnicity, or body composition [12]. As these values are understood to fluctuate 
among temporal, genetic, and anthropometric subpopulations, indiscriminate reliance on these reference estimates presents a known source of error for HW [5], and this technique has come to be supplanted by combinatory multi-component models as the criterion laboratory determination of in vivo body composition $[13,14]$.

DXA is an expedient, non-invasive, and relatively novel technology that has been considered a valid and reliable reference method for BCA by some researchers [15, 16, 17, 7]. Other experts, however, have taken issue with the manifest limitations of DXA, including minor systematic errors associated with altered hydration status $[18,19]$ methodological difficulty in parsing overlapping regions of bone and soft tissue [20], a tendency to significantly under-predict $\% \mathrm{BF}$ in lean subjects [21, 22 ], and signal attenuation with sagittal abdominal diameter $>$ $20 \mathrm{~cm}$ leading to diminished accuracy in obese populations [2325]. These issues are in addition to broad inability to directly compare DXA outcomes from disparate DXA instruments, even those from the same manufacturer, due to proprietary predictive algorithms and other non-trivial performance disparities $[26,27]$. Moreover, DXA presents many disadvantages more traditionally associated with complex laboratory measures, such as prohibitive cost, immobility, limited availability, and the need for certified operators [26].

Due to the established limitations of both DXA and HW, a popular field alternative for assessing body composition is BIA, which is often favored due to its relative affordability, portability, and non-invasive nature $[6,28]$. In essence, BIA estimates body volume from a given length and measured resistance to electrical current, relying on four primary assumptions with understood limitations: (1) the human body is shaped like a perfect cylinder of uniform length and cross-sectional area, (2) impedance to electrical conductance through the human body is directly related to the length of the conductor and inversely related to its cross sectional area, (3) impedance is a function of resistance and reactance, and (4) human tissues act discretely as either conductors (muscle mass), or insulators (fat mass), with the current following the path of least resistance [28].

Tissue electrical impedance can be measured using either Single Frequency (SF) devices, able to assess only extracellular fluid, or Multi-Frequency (MF) devices, which estimate fluid volume in both intra- and extracellular compartments [28]. Numerous BIA devices have been commercially developed, with the efficacy of these devices in the general population typically being established through comparison to reference measures such as HW and DXA [29]. Currently, there is lack of data validating SF-BIA and MF-BIA performance in overweight or obese individuals using criterion measures such as HW or DXA, with only a handful of published articles presently available $[16,30,31]$.

Excess body fat is the fundamental root of obesity and associated health concerns. Consequently, a simple, expedient and dependable assessment of body composition within overweight and obese populations is particularly desirable, both for accurate single evaluation of acute condition, and for valid comparison of repeated measures while monitoring weight fluctuations over time [2, 32]. Unfortunately, some laboratory methods, such as ADP, are simply methodologically unsuitable for obese populations, while data illuminating the most widely used method for these overweight subjects, BIA, is limited $[32,33]$. Accordingly, the purpose of this study was to compare the performance of two separate BIA devices, the Omron HBF 306 (SF-BIA) and Impedimed SFB7 (MF-BIA), in overweight and obese young adults, using both DXA and HW as comparative reference measures.

\section{Materials and Methods}

\section{Subjects}

The protocol utilized by this study was approved in advance by the Institutional Review Board for human subjects at California State University - San Bernardino. Twenty-five overweight or obese adults (BMI $\geq 25 \mathrm{~kg} / \mathrm{m}^{2}$ ) volunteered to participate in this project, with written informed consent obtained from each participant prior to data collection. Prospective participants were excluded from the investigation if they presented with a serious medical condition such as heart disease, diabetes, renal disease, or cancer; had a pacemaker; were currently pregnant or breastfeeding; had a body weight $>350$ pounds, or reported currently using medications or dietary supplements understood to impact body composition (i.e. ephedra, capsaicin, caffeine, thermogenic compounds, or any herbal or botanical purported to facilitate weight loss). Eligible participants were instructed to arrive euhydrated for data collection, and to refrain from alcohol use for 48 hours, strenuous exercise for 24 hours, and nutrient consumption for 4 hours prior to all testing.

\section{Research Protocol}

All measurements were collected from each participant during a single testing session lasting approximately 3 hours. Upon arrival at the laboratory, all participants were initially asked to void their bladder, if possible. This was immediately followed by assessment of participants' barefoot body mass and standing height (Dectecto Cardinal Scale Manufacturing Co., Webb City, MO, USA); each taken as the average of duplicate measurements, and measured to the nearest $0.01 \mathrm{~kg}$ and $0.1 \mathrm{~cm}$, respectively. After completing these initial assessments, participants then rotated through the following stations in no specific order: SFBIA (Omron Model HBF-306, Omron Healthcare, Vernon Hills, IL, USA); MF-BIA (ImpediMed SFB7, Impedimed, Ltd., Eight Mile Plains, Queensland, Australia); DXA (enCORE 2010; software v.13.50.040; GE/Lunar Corp, Madison, WI, USA); and HW (VacuMed Ventura, CA).

\section{Omron HBF-306 BIA (SF-BIA)}

Each participant's sex, height, weight, and age in years were entered into the SF-BIA device. Height was entered to the nearest $0.25 \mathrm{in}$, while weight was entered to the nearest $0.5 \mathrm{lb}$. Participants were instructed to stand upright, with feet shoulderwidth apart, arms parallel to the ground, and elbows extended. They were then asked to grasp the handles and maintain contact 
with the metal sensor electrodes for approximately 5 seconds, until the process was completed.

\section{ImpediMed SFB7 (MF-BIA)}

Participants were instructed to lay supine on a table with arms and legs abducted $30-45^{\circ}$ for five minutes. Prior to electrode placement, excess body hair was removed, and the bare skin at each site was cleaned with alcohol. Next, height, weight, age, and sex for each subject was entered into the MF-BIA device, with electrodes then placed at the right hand and foot in accordance with manufacturer recommendations. Hydration was assessed using the Total Body Water (TBW) values generated by the MFBIA device via Bioelectrical Impedance Spectroscopy (BIS). All subjects were within the desirable range for TBW values for adequate hydration status [34].

\section{Dual-energy X-ray absorptiometry (DXA)}

All DXA measurements were performed by a licensed operator. The $\mathrm{GE}^{\circledR}$ Prodigy bone densitometer (enCORE 2010; software v.13.50.040) utilized for this study was calibrated once daily according to manufacturer specifications. Prior to measurement, subjects were asked to wear light athletic type clothing which did not include metal zippers, metal underwire bras, and to remove belts and/or jewelry. Participants were then instructed to lay supine and remain motionless on the scanning bed for the duration of the whole body scan.

\section{Hydrostatic Weighing (HW)}

Body density was evaluated from HW, corrected for Residual Lung Volume (RV). RV was determined on land with the participant in a seated position, using the oxygen dilution method [35], as assessed by metabolic cart (True One 2400®, Parvo-Medics, Inc. Provo, Utah). In order to be considered a valid RV measurement, a minimum of two trials were obtained, with an average of the nearest two trials within $\pm 5 \%$ recorded as the final measure. Following RV assessment, participants obtained a dry body weight using a Dectecto ${ }^{\circledR}$ weight scale (Dectecto Cardinal Scale Manufacturing Co., Webb City, MO, USA) while wearing only the garments used during subsequent $\mathrm{HW}$, and then asked to shower in order to remove any commercial products, oils, and other accumulations from the skin prior to submersion. Participants then entered the HW tank, and were seated in a metal swing seat suspended from a calibrated Lode Cell (Loadcell No. 17062, VacuMed, Ventura, CA). Each participant was instructed to submerge their entire body, and to then perform a maximum voluntary exhalation underwater, within \pm of $100 \mathrm{~g}$ where the average of the three highest values within 6-10 trials was used as the final determination of underwater weight.

\section{Statistical Analysis}

We employed separate 2-way repeated-measures analyses of variance (RM-ANOVA) to compare mean outcomes (as \%BF) obtained from 4 separate BCA methods (SF-BIA, MF-BIA, DXA, and $\mathrm{HW}$ ) for the entire sample, as well as divided according to sex (male and female). This procedure was accompanied by post hoc group-wise comparison of adjusted means, using the
Bonferroni correction to adjust for multiple comparisons, as appropriate. Bland and Altman (1986) plots showing limits of agreement were created for all pairings of SF-BIA, MF-BIA, DXA, and $\mathrm{HW}$, with values expressed as mean difference, Lower Limits Of Agreement (LLA), and Upper Limits Of Agreement (ULA). All statistical analyses were performed using SPSS version 22 (IBM, Chicago, IL, 2013) with statistical significance for all tests set $a$ priori at $p \leq 0.05$.

\section{Results}

Subject demographic information for the complete sample, as well as data parsed by sex, and mean \%BF estimations for each method, are displayed in Table 1.

There was a significant between-subjects effect between all BCA modalities for the group $F(1,23)=63.8, p<0.001)$. Additionally, there was a significant within-subjects effect $F$ (1.6, $31.1)=17.078, p<0.001)$. A significant difference was detected between DXA and HW in mean estimation of \%BF $(p<0.001)$. When DXA results were compared to both the SF-BIA and MF-BIA techniques, a significant difference was also observed between average DXA and SF-BIA outcomes $(p<0.001)$. Likewise, when the SF-BIA and MF-BIA techniques were compared, there was a significant difference in mean estimation of \%BF ( $p=0.015)$. There was also a strong correlation between SF-BIA and HW outcomes ( $r=0.902, p<0.05$ ), as well as a lesser, though significant, correlation between DXA and MF-BIA results ( $r=$ $0.709, \mathrm{p}<0.05$ ). The agreement between methods for estimation of \%BF is summarized in Bland-Altman plots (Figures 1 and 2).

\section{Discussion}

Overall, the results of this project help demonstrate that, in young adults who are overweight or obese, different methods for estimating \%BF provide dissimilar results. These outcomes depict large individual variability in estimations of $\% \mathrm{BF}$, with significant differences apparent between the putative reference

Table 1: Demographics of study participants. DXA: Dual Energy X-Ray Absorptiometry; MF-BIA: Multi-frequency Bioelectrical Impedance analysis; SF-BIA: Single frequency bioelectrical impedance analysis; TBW: total body water; UW: underwater weighing; \% BF: Percent body fat; \% BW: Percent body weight

\begin{tabular}{|c|c|c|c|c|c|c|}
\hline \multirow[b]{2}{*}{ Parameter } & \multicolumn{2}{|c|}{ Total $(\mathrm{N}=25)$} & \multicolumn{2}{|c|}{ Male $(n=13)$} & \multicolumn{2}{|c|}{ Female $(n=12)$} \\
\hline & $\mathbf{M}$ & SD & M & SD & M & SD \\
\hline Age (Year) & 24.6 & 4.7 & 26.2 & 5.9 & 22.8 & 1.7 \\
\hline Height (cm) & 160.9 & 39.3 & 165.3 & 49.8 & 156.2 & 24.7 \\
\hline Weight (kg) & 82.9 & 18.7 & 86.4 & 18.9 & 79.2 & 18.5 \\
\hline $\begin{array}{l}\text { Body Mass Index } \\
\left(\mathrm{kg} / \mathrm{m}^{2}\right)\end{array}$ & 30.1 & 4.1 & 29.4 & 3.6 & 30.8 & 4.6 \\
\hline DXA (\% BF) & 34.2 & 10.6 & 25.5 & 6.6 & 43.0 & 5.3 \\
\hline HW (\% BF) & 28.2 & 9.3 & 20.8 & 6.1 & 35.7 & 5.3 \\
\hline SF-BIA (\% BF) & 26.2 & 8.0 & 20.1 & 5.7 & 32.9 & 3.5 \\
\hline MF-BIA (\% BF) & 30.9 & 8.8 & 25.1 & 7.1 & 37.3 & 5.3 \\
\hline TBW (\% BW) & 51.4 & 18.2 & 57.2 & 23.7 & 45.2 & 5.5 \\
\hline
\end{tabular}


methods DXA and HW, as well as between the commonlyemployed SF- and MF-BIA techniques.

At present, DXA is becoming increasingly popular for the assessment of soft tissue composition, in addition to the evaluation of bone mineral density, for which DXA was originally designed [8]. While the rapid, noninvasive nature of DXA is understandably attractive to many patients, this method also presents numerous non-trivial limitations that have caused some experts to question whether DXA provides sufficient accuracy and precision of measurement to serve as a scientific "gold standard" [36].

Importantly for overweight and obese populations, DXA's estimates of bone mineral content and soft tissue composition are sensitive to anteroposterior thickness of the body [36, 37]. Additionally, DXA estimation errors increase significantly in regard to pixels containing bone, raising concern for the ability of the DXA to accurately distinguish soft tissue in the thorax [20,

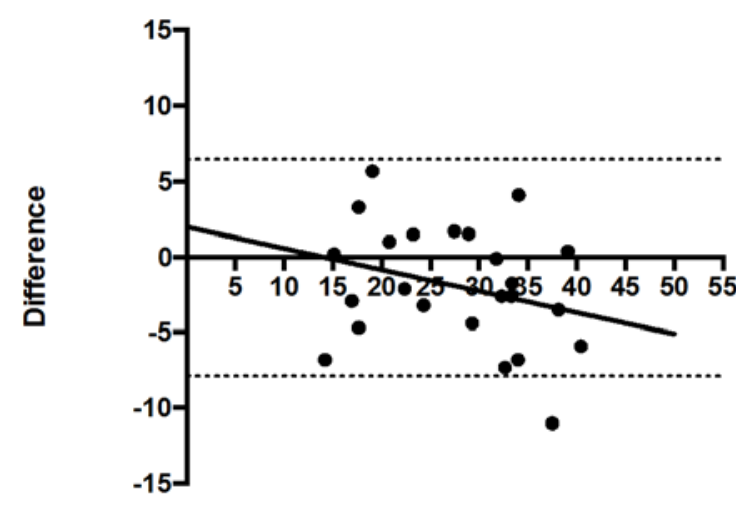

Average

Figure 1: Plot of differences for SF-BIA and HW. The mean difference and 95\% limits of agreement are indicated

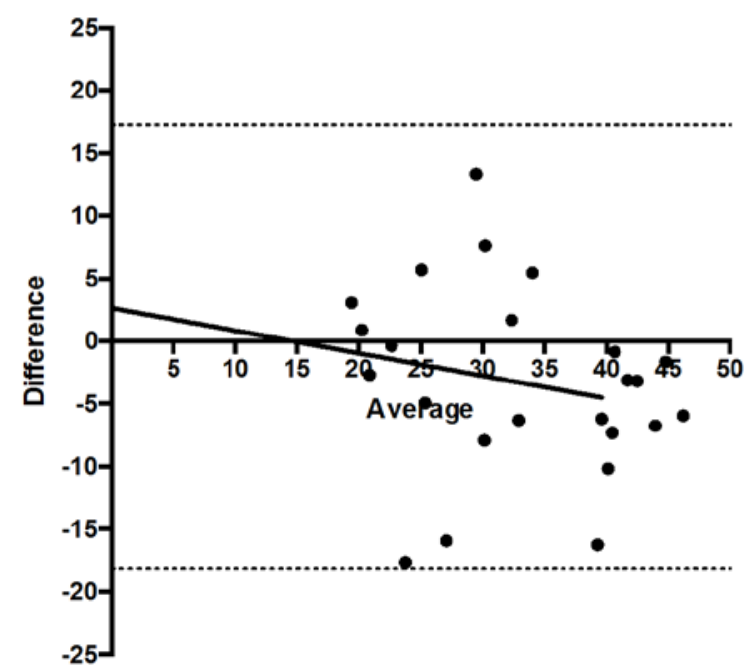

Figure 2: Plot of differences for MF-BIA and DXA. The mean difference and $95 \%$ limits of agreement are indicated
8]. This is a point of some importance for overweight individuals, as excess body fat often accumulates in substantial stores surrounding the trunk $[33,36]$. In this region, the arrangement of the ribs and spine prevents the DXA beam from directly identifying many areas of soft tissue, these requiring predictive estimation from adjacent pixels, which can lead to significant overestimation of \% BF [36]. This methodological bias may help explain the results of the present investigation, which found that DXA determinations overestimated body fat by an average of $6 \%$ for the entire study population when compared to HW outcomes $(p<0.05)$, with a $4.7 \%$ mean overestimation of body fat for males, and a mean $7.2 \%$ overestimation of body fat for females. This degree of deviation between so-called 'criterion' methods in an overweight population raises concern when using DXA BCA to monitor overweight subjects, particularly during periods of intended weight-reduction.

When mean DXA and HW outcomes were compared to average results from SF-BIA and MF-BIA, a significant difference was detected $(p<0.05)$ between the $\%$ BF estimates produced by DXA and SF-BIA, though HW results were not statistically different $(p \geq 0.05)$ on average from SF-BIA or MF-BIA. While the SF-BIA was found to significantly underestimate $\% \mathrm{BF}$ relative to mean DXA outcomes, SF-BIA results were significantly correlated with HW ( $p<0.05, r=0.902)$, lending credence to their veracity. This outcome builds agreement with other projects investigating BCA in obese individuals; these researchers, which have previously warned that DXA and SF-BIA devices cannot be used interchangeably in light of the additive variability encompassed by these methods [38-40].

While the strong agreement with HW suggests that SF-BIA measures observed here are dependable, it can be argued the apparent disparity observed between average SF-BIA and DXA outcomes may be at least partially explained by the methodological underpinnings of SF-BIA technology. Single frequency devices, including the SF-BIA utilized herein, operate only at $50 \mathrm{kHz}$, a low frequency current unable to penetrate cellular membranes to allow determination of intracellular fluid, ultimately promoting a bias toward underestimation of \%BF $[41,28]$. Moreover, the assessment of tissue impedance performed by the SF-BIA device used in this project is restricted to the upper body, which may exacerbate differences observed between males and females, or between various ethnic populations [42, 43]. Perhaps most importantly, the equations employed by this SF-BIA device may also have plausibly contributed to an underestimation of $\% \mathrm{BF}$, as these models were developed using normal-weight populations [20].

Interestingly, DXA and MF-BIA estimates of \%BF recorded in this study were also found to be significantly correlated ( $p<$ $0.05, r=0.709$ ), though these measures were significantly higher $(p<0.05)$ on average than HW or SF-BIA, respectively. This methodological disparity has important ramifications for BCA in this population, and is reflected elsewhere in the literature. Another contemporary project reported that MF-BIA showed better agreement with DXA estimations of body composition than SF-BIA in post-menopausal women (BMI: $26.6 \pm 4.1 \mathrm{~kg} / \mathrm{m}^{2}$ ), 
with the latter technique found to produce significantly lower determinations of \%BF in comparison to DXA [44]. Similarly, one recent study reported strong agreement between MF-BIA and DXA estimates of body composition in obese clinical patients [45], while another reported that SF-BIA tended to underestimate $\% \mathrm{BF}$ compared to DXA [46]. Importantly, the latter project reported that the magnitude of SF-BIA disagreement with DXA increased in proportion to increasing $\% \mathrm{BF}$, with the bias significantly elevated for females [46].

None of these projects utilized another comparative reference measure to verify DXA performance in overweight subjects, while the results of the current study suggest that DXA cannot be expected to perform similarly to HW, an established "gold standard" [43], and may not be suitable as a criterion measure in this population. Clearly, this outcome complicates interpretation of existing body composition research in subjects with excess body fat, and these systematic disparities in BCA require further research to illuminate the best possible techniques for assessing overweight subjects.

The limitations of this study are comparable to a preponderance of acute body composition research. Firstly, our participants had a maximum weight threshold of $130 \mathrm{~kg}$, this due to the weight restrictions of the DXA apparatus. As a result, these conclusions may not generalize to individuals who weigh $>130$ kg. Additionally, BIA, DXA, and HW methodologies each assume that the hydration of FFM is constant between individuals (73.2 $\%$ ), while subjects herein were measured in a randomized order during a single prolonged session without access to food or water. Moreover, as subjects were measured at only a single time point, the results of this study lack direct assessment of measurement reliability. The current study was also unable to directly assess the hydration status of subjects prior to testing. To mitigate this inability, and attempt to standardize hydration of the study participants, written pre-testing guidelines were issued to subjects in advance of participation, then discussed with the individual directly two days prior to participation.

In conclusion, the present study adds to a limited body of research examining the accuracy of BCA techniques in overweight populations. It is the authors' hope that the findings of this study assist in the identification of BCA methods most suitable for overweight or obese individuals, particularly in regard to the putative 'criterion' methods applied to this population. The results of the current project indicate that DXA and HW cannot be used interchangeably in overweight and obese subjects, and suggest that significant differences exist between SF- and MF-BIA techniques in these individuals. Our data also indicate that SF-BIA performance strongly correlates with HW in subjects with excess body fat; however, these methods both produced significantly different estimates of $\% \mathrm{BF}$ on average than DXA, suggesting suboptimal DXA performance in this group. Interestingly, MF-BIA devices were found to generate similar \% BF estimations to DXA on average, and the efficacy of these devices in overweight and obese subjects may warrant further investigation. Finally, future studies utilizing substantial sample sizes are requisite in order to robustly demonstrate the performance of available BCA techniques across an expanded range of human body composition, and to help ensure accurate analysis of body composition in those subjects for whom these measures are perhaps most clinically consequential.

\section{Conflict of Interest}

The authors declare that there is no potential conflict of interest regarding the publication of this paper.

\section{References}

1. Ogden CL, Carroll MD, Kit BK, Flegal KM. Prevalence of Childhood and Adult Obesity in the United States, 2011-2012. JAMA. 2014;311(8):80614. doi: 10.1001/jama.2014.732.

2. Pourhassan M, Schautz B, Braun W, Gluer CC, Bosy-Westphal A, Müller MJ. Impact of body-composition methodology on the composition of weight loss and weight gain. Eur J Clin Nutr. 2013;67(5):446-54. doi: 10.1038/ejcn.2013.35.

3. Johnson KE, Naccarato IA, Corder MA, Repovich WE. Validation of three body composition techniques with a comparison of ultrasound abdominal fat depths against an octopolar bioelectrical impedance device. Int J Exerc Sci. 2012;5(3):205-213.

4. Pineau JC, Guihard-Costa AM, Bocquet M. Validation of ultrasound techniques applied to body fat measurement. A comparison between ultrasound techniques, air displacement plethysmography and bioelectrical impedance vs. dual-energy X-ray absorptiometry. Ann Nutr Metab. 2007;51(5):421-7.

5. Wagner DR, Heyward VH. Techniques of body composition assessment: a review of laboratory and field methods. Res Q Exerc Sport. 1999;70(2):135-49.

6. Ellis KJ. Human body composition: in vivo methods. Physiol Rev. 2000;80(2):649-80.

7. Prior BM, Cureton KJ, Modlesky CM, Evans EM, Sloniger MA, Saunders $\mathrm{M}$, et al. In vivo validation of whole body composition estimates from dual-energy X-ray absorptiometry. J Appl Physiol. 1997;83(2):623-30.

8. Ackland TR, Lohman TG, Sundgot-Borgen J, Maughan RJ, Meyer NL, Stewart $\mathrm{AD}$, et al. Current status of body composition assessment in sport: review and position statement on behalf of the ad hoc research working group on body composition health and performance, under the auspices of the I.O.C. Medical Commission. Sports Med. 2012;42(3):227-49. doi: 10.2165/11597140-000000000-00000.

9. Pace N, Rathbun EN. Studies on body composition III. The body water and chemically combined nitrogen content in relation to fat content. Journal of Biological Chemistry. 1945.

10. Keys A, Brozek J. Body Fat in Adult Man. Physiol Rev. 1953;33(3):245325 .

11. Behnke AR, Feen BG, Welham WC. The Specific Gravity of Healthy Men. Journal of the American Medical Association. 1942;118(7):495-498.

12. Brozek J, Grande F, Anderson JT, Keys A. Densitometric Analysis of Body Composition: Revision of Some Quantitative Assumptions. Ann N Y Acad Sci. 1963;110:113-40.

13. Friedl KE, DeLuca JP, Marchitelli LJ, Vogel JA. Reliability of bodyfat estimations from a four-compartment model by using density, body water, and bone mineral measurements. Am J Clin Nutr. 1992;55(4):764-70.

14. Wilson JP, Strauss BJ, Fan B, Duewer FW, Shepherd JA. Improved 4-compartment body-composition model for a clinically accessible 
measure of total body protein. Am J Clin Nutr. 2013;97(3):497-504. doi: 10.3945/ajcn.112.048074.

15. Kaul S, Rothney MP, Peters DM, Wacker WK, Davis CE, Shapiro MD, et al. Dual-energy X-ray absorptiometry for quantification of visceral fat. Obesity (Silver Spring). 2012;20(6):1313-8. doi: 10.1038/ oby.2011.393.

16. Pateyjohns IR, Brinkworth, GD, Buckley JD, Noakes M, Clifton PM. Comparison of three bioelectrical impedance methods with DXA in overweight and obese men. Obesity (Silver Spring). 2006;14(11):206470.

17. Sun G, French CR, Martin GR, Younghusband B, Green RC, Xie YG, et al. Comparison of multifrequency bioelectrical impedance analysis with dual-energy X-ray absorptiometry for assessment of percentage body fat in a large, healthy population. Am J Clin Nutr. 2005;81(1):74-8.

18. St-Onge MP, Wang Z, Horlick M, Wang J, Heymsfield SB. Dual-energy $\mathrm{X}$-ray absorptiometry lean soft tissue hydration: independent contributions of intra- and extracellular water. Am J Physiol Endocrinol Metab. 2004;287(5):E842-7.

19. Pietrobelli A, Wang Z, Formica C, Heymsfield SB. Dual-energy X-ray absorptiometry: fat estimation errors due to variation in soft tissue hydration. Am J Physiol. 1998;274(5 Pt 1):E808-16.

20. Pietrobelli A, Formica C, Wang Z, Heymsfield SB. Dual-energy X-ray absorptiometry body composition model: review of physical concepts. Am J Physiol. 1996;271(6 Pt 1):E941-51.

21.Van der Ploeg GE, Withers RT, Laforgia J. Percent body fat via DEXA: comparison with a four-compartment model. J Appl Physiol. 2003;94(2):499-506

22. Fields DA, Goran MI. Body composition techniques and the fourcompartment model in children. J Appl Physiol. 2000;89(2):613-20.

23. Laddu DR, Lee VR, Blew RM, Sato T, Lohman TG, Going SB. Predicting visceral adipose tissue by MRI using DXA and anthropometry in adolescents and young adults. Int J Body Compos Res. 2012;10(4):93100.

24.Goodsitt MM. Evaluation of a new set of calibration standards for the measurement of fat content via DPA and DXA. Med Phys. 1992;19(1):35-44.

25. LaForgia J, Dollman J, Dale MJ, Withers RT, Hill AM. Validation of DXA Body Composition Estimates in Obese Men and Women. Obesity (Silver Spring). 2009;17(4):821-6. doi: 10.1038/oby.2008.595.

26. Nana A, Slater GJ, Stewart AD, Burke LM. Methodology review: using dual-energy X-ray absorptiometry (DXA) for the assessment of body composition in athletes and active people. Int J Sport Nutr Exerc Metab. 2015;25(2):198-215. doi: 10.1123/ijsnem.2013-0228.

27. Lohman M, Tallroth K, Kettunen JA, Marttinen MT. Reproducibility of dual-energy x-ray absorptiometry total and regional body composition measurements using different scanning positions and definitions of regions. Metabolism. 2009:58(11):1663-8. doi: 10.1016/j.metabol.2009.05.023.

28. Kyle UG, Bosaeus I, De Lorenzo AD, Deurenberg P, Elia M, Gómez JM, et al. Bioelectrical impedance analysis-part I: review of principles and methods. Clin Nutr. 2004;23(5):1226-43.

29. Demura S, Sato S, Kitabayashi T. Percentage of total body fat as estimated by three automatic bioelectrical impedance analyzers. ] Physiol Anthropol Appl Human Sci. 2004;23(3):93-9.

30. Jakicic JM, Wing RR, Lang W. Bioelectrical impedance analysis to assess body composition in obese adult women: the effect of ethnicity. Int J Obes Relat Metab Disord. 1998;22(3):243-9.
31. Thomson R, Brinkworth GD, Buckley JD, Noakes M, Clifton PM. Good agreement between bioelectrical impedance and dual-energy X-ray absorptiometry for estimating changes in body composition during weight loss in overweight young women. Clin Nutr. 2007;26(6):771-7.

32. Das SK, Roberts SB, Kehayias JJ, Wang J, Hsu LK, Shikora SA, Saltzman E, McCrory MA. Body composition assessment in extreme obesity and after massive weight loss induced by gastric bypass surgery. Am J Physiol Endocrinol Metab. 2003;284(6):E1080-8.

33. Ritz P, Sallé A, Audran M, Rohmer V. Comparison of different methods to assess body composition of weight loss in obese and diabetic patients. Diabetes Res Clin Pract. 2007;77(3):405-11.

34. O'Brien C, Young AJ, Sawka MN. Bioelectrical impedance to estimate changes in hydration status. Int J Sports Med. 2002;23(5):361-6.

35. Wilmore JH, Vodak PA, Parr RB, Girandola RN, Billing JE. Further simplification of a method for determination of residual lung volume. Med Sci Sports Exerc. 1980;12(3):216-8.

36. Roubenoff R, Kehayias JJ, Dawson-Hughes B, Heymsfield SB. Use of dual-energy x-ray absorptiometry in body-composition studies: not yet a "gold standard". Am J Clin Nutr. 1993;58(5):589-91.

37. Jebb SA. Measurement of soft tissue composition by dual energy X-ray absorptiometry. Br J Nutr. 1997;77(2):151-63.

38. Jiménez A, Omaña W, Flores L, Coves MJ, Bellido D, Perea V, et al. Prediction of Whole-Body and Segmental Body Composition by Bioelectrical Impedance in Morbidly Obese Subjects. Obes Surg. 2012;22(4):587-93.

39. Berstad P, Randby A, Seim Ekeland G, Ulveland H, Omland T, Almendingen K. Body fat and fat-free mass measured by bioelectric impedance spectroscopy and dual-energy X-ray absorptiometry in obese and non-obese adults. Br J Nutr. 2012;107(8):1192-200. doi: 10.1017/S000711451100417X.

40. Bedogni G, Agosti F, De Col A, Marazzi N, Tagliaferri A, Sartorio A. Comparison of dual-energy X-ray absorptiometry, air displacement plethysmography and bioelectrical impedance analysis for the assessment of body composition in morbidly obese women. Eur J Clin Nutr. 2013;67(11):1129-32. doi: 10.1038/ejcn.2013.159.

41. De Lorenzo A, Andreoli A, Deurenberg P. Impedance Ratio as a Measure of Water Shifts. Annals of Nutrition and Metabolism. 1997;41(1):2228.

42. Dehghan M, Merchant AT. Is bioelectrical impedance accurate for use in large epidemiological studies? Nutr J. 2008;7:26. doi: 10.1186/1475-2891-7-26.

43. Wagner DR, Heyward VH. Measures of body composition in blacks and whites: a comparative review. The American journal of clinical nutrition. 2000;71(6),1392-1402.

44.Gába A, Kapuš O, Cuberek R, Botek M. Comparison of multi- and single-frequency bioelectrical impedance analysis with dual-energy $\mathrm{X}$-ray absorptiometry for assessment of body composition in postmenopausal women: effects of body mass index and accelerometerdetermined physical activity. Journal of Human Nutrition and Dietetics. 2014; 28(4):390-400.

45. Faria SL, Faria OP, Cardeal MD, Ito MK. Validation Study of MultiFrequency Bioelectrical Impedance with Dual-Energy X-ray Absorptiometry Among Obese Patients. Obes Surg. 2014;24(9):147680. doi: $10.1007 / \mathrm{s} 11695-014-1190-5$.

46. Li YC, Li CI, Lin WY, Liu CS, Hsu HS, Lee CC, et al. Percentage of Body Fat Assessment Using Bioelectrical Impedance Analysis and DualEnergy X-ray Absorptiometry in a Weight Loss Program for Obese or Overweight Chinese Adults. PLoS One. 2013;8(4):e58272. doi: 10.1371/journal.pone.0058272. 